Peer-Reviewed Case Series

\title{
Restoration Of Left Ventricular Function Following LVAD Implantation - Remission Or Recovery? A Case series
}

Citation: Joshi S. et al. (2018)

"Restoration Of Left Ventricular

Function Following LVAD

Implantation - Remission Or

Recovery? A Case series".

The VAD Journal, 4. doi: https://doi.org/10.13023/vad.2018 .12

Editor-in-Chief: Maya Guglin, University of Kentucky

Received: July 23, 2018

Accepted: November 2, 2018

Published: November 3, 2018

() 2018 The Author(s). This is an open access article published under the terms of the Creative Commons Attribution-

NonCommercial 4.0 International License

(https://creativecommons.org/lice nses/by-nc/4.0/), which permits unrestricted non-commercial use, distribution, and reproduction in any medium, provided that the original author(s) and the publication source are credited.

Funding: Not applicable

Competing interests:Not applicable

\section{Shiksha Joshi, Mariah Barlow*, and Maya Guglin}

*Corresponding author: mariah.barlow@uky.edu

University of Kentucky, Lexington, KY

\section{Abstract}

\section{Background}

A wide range of left ventricular (LV) recovery rates on left ventricular assist device (LVAD) support have been reported. In this case series, we summarize our experience with LVAD explantation for presumed LV recovery.

\section{Case series}

Out of 240 patients who received an LVAD implant in our program since its inception, we explanted/inactivated the pump for presumed LV recovery in only three patients. All three of these patients had relapse of cardiomyopathy within 6 months of explantation.

\section{Conclusion}

Our experience does not support the existence of LV recovery following LVAD implantation as a common phenomenon. Improvement in LV function on LVAD is sustainable only with mechanical circulatory support and once the offloading support provided by the device is removed recurrent failure quickly ensue. Likely the term "remission" reflects the status more accurately than the term "recovery".

Keywords: left ventricular assist device, heart failure, recovery 


\section{Introduction}

Since the publication of the famous paper by Birks et al. in 2006 reporting a stunning $73 \%$ prevalence of left ventricular (LV) recovery allowing explantation of previously implanted pulsatile-flow left ventricular assist devices (LVADs) with no recurrence of heart failure (HF), LV recovery has continued to be a controversial topic (1). The magnitude of recovery was first ascribed to clenubetrol, which they used in high doses to facilitate myocardial strength and contraction. Following this, in 2011, a similar study was done with a non-pulsatile LVAD (HeartMate II, Thoratec, Pleasanton, CA), without clenubetrol, and still demonstrated a $60 \%$ recovery rate (2). Meanwhile however, data from INTERMACS demonstrated LV recovery allowing explantation in only $0.9 \%$ at 1 -year, $1.9 \%$ at 2 -year, and $3.1 \%$ at 3-year follow-up (3). Given the varied data available there is on-going debate regarding the true prevalence of LV recovery following LVAD implantation.

In our center. over 250 patients have been implanted with long-term LVADs. Only three patients demonstrated the degree of recovery sufficient for explantation of their pumps, but afterward all went on to develop recurrent LV failure. We are presenting this case series and questioning the durability of myocardial recovery resulting from the mechanical unloading on LVAD.

\section{Case 1}

A 55 year old male with a 10 year history of systolic heart failure secondary to a non-ischemic cardiomyopathy presented with progressive New York Heart Association (NYHA) functional class IV symptoms. At time of presentation he was on guideline directed medical therapy and had an implantable Cardioverterdefibrillator (ICD) in place. The electrocardiogram (ECG) demonstrated normal sinus rhythm with no bundle branch block and a normal QRS duration. $\mathrm{He}$ underwent placement of a Heartmate II LVAD (Thoratec, Pleasanton, CA). His preimplant echo showed an LVEF of $13 \%$. After receiving the implant, he was regularly followed up in LVAD clinic. He continued to be treated with a beta blocker and angiotensine converting enzyme (ACE) inhibitor while his LVAD was in place and had drastic improvement in his heart failure symptoms. An echocardiogram was repeated 3 months after LVAD implantation which demonstrated an LVEF of $25-35 \%$, which was the first evidence of some degree of myocardial recovery. One and a half years following device implantation he presented with altered mental status and was ultimately diagnosed with streptococcal pneumonia. A computer tomography (CT) of the chest demonstrated several abscesses within the LVAD pocket. Irrigation and drainage of the abscesses were performed, however the device itself was grossly infected. Perioperative echo with ramp study showed further improvement in LV function with LVEF of $40-45 \%$. Given the patient demonstrated improvement in his ejection fraction and because the device was grossly infected, the device was removed. He was treated with appropriate antibiotics with clinical improvement and was discharged home with continued guideline directed medical therapy. Six months later, he presented with symptoms of heart failure, and his LVEF has declined to $30 \%$ at that time. Another 6 months later (total of 12 months post-explantation), a repeat echocardiogram 
demonstrated that his LVEF had returned to less than $20 \%$. He was admitted multiple times for acute decompensated heart failure exacerbations and eventually became inotrope dependent. He was not a candidate for repeat LVAD due to extensive adhesions and social limitations. Ultimately, he was referred to hospice care.

\section{Case 2}

A 48 year old male presented with 6 months of progressive dyspnea on exertion. He was previously healthy with no past medical history. A transthoracic echo showed an LVEF $15 \%$ with no significant valvular abnormalities. He underwent coronary angiography which showed no obstructive coronary artery disease. He was started on guideline directed medical therapy titrated to maximally tolerated dosages and participated in a cardiac rehabilitation program. Despite appropriate medical therapy, he had on-going NYHA class IV heart failure symptoms, was placed on continuous inotropic therapy and then ultimately underwent placement of a Heartmate II LVAD. He continued to be treated with an ACE inhibitor and beta blocker. In 9 months after implantation an echocardiogram revealed an LVEF of 25 to $35 \%$. At 1 year post implantation, an echocardiogram was repeated, now demonstrating an LVEF of greater than 55\%. After weaning of the pump (8600 revolutions per minute (rpms) to $6000 \mathrm{rpms}$ ), serial echocardiograms continued to show an intact LVEF of 58 to $61 \%$ with normal LV end diastole internal dimension at $3.7-3.9 \mathrm{~cm}$ indicating the possibility of LV recovery. Right heart catheterization was performed, and it confirmed normal right and left heart filling pressures despite reduction of the LVAD speed to 6000 rpms. Given evidence of LV recovery, the device was explanted 15 months following implantation. Following device removal he was continued on guideline directed medical therapy at the maximally tolerated dosages. While he initially remained stable following explantation, within the first year he developed recurrent symptoms accompanied by a gradual decline in LV function. First evidence of recurrent LV failure occurred at 3 months post-explantation at which time LVEF has declined to $35 \%$. The ejection fraction remained stable for the next 9 months before ultimately declining to around 20 to $25 \%$ one year after the device had been removed. At this time, he currently remains on heart failure medications and has not yet required reimplantation of a LVAD or initiation of inotropic therapy.

\section{Case 3}

A 50 year old female with a 30 year history of systolic heart failure secondary to peripartum cardiomyopathy presented with progressing NYHA IV symptoms. At the time of presentation she was on guideline directed medical therapy, had undergone cardiac rehabilitation therapy, and had an ICD in place. An ECG demonstrated normal sinus rhythm. An echocardiogram was performed which demonstrated and LVEF of less than $20 \%$, along with severe LV dilatation. As she was not eligible for transplant due to obesity and recent smoking, a Heartmate II LVAD was placed. She did well following device implantation, was continued on goal directed medical therapy, continued to participate in cardiac rehabilitation and was followed routinely in LVAD clinic. In subsequent months, her LVEF recovered 
to 50 to $55 \%$. The pump speed was serially reduced from 9200 rpms to 8400 rpms, and then further to $6000 \mathrm{rpms}$, and all the while her LVEF remained stable at 50 to $55 \%$. An LVAD recovery study was performed which showed normal LV size and function with normal intra-cardiac filling pressures on right heart catheterization while on minimal pump speed. With the swan-ganz catheter left in, she remained with normal hemodynamics for 24 hours on minimal pump speed, with pulmonary capillary wedge pressures staying at $12 \mathrm{mmHg}$. 19 months after implantation, the LVAD was inactivated (because of technical difficulty and intraoperative bleeding, the pump was left inside with the outflow graft stapled closed). She was managed on appropriate medical therapy. By 3 months following device inactivation, her LVEF had returned to less than $20 \%$ with recurrent severe global hypokinesis and severe LV dilatation. She was initiated on inotropic support with milrinone. At present, she remains on continuous home inotropic therapy.

The dynamic changes of echocardiographic parameters of all three patients are presented in Table 1.

Table 1. The dynamic changes of echocardiographic parameters

\begin{tabular}{|c|c|c|c|c|c|c|c|}
\hline & & $\begin{array}{l}\text { pre- } \\
\text { LVAD }\end{array}$ & $\begin{array}{l}\text { on } \\
\text { LVAD }\end{array}$ & \multicolumn{4}{|c|}{ Post-LVAD } \\
\hline & & & & $\begin{array}{l}1 \\
\text { week }\end{array}$ & $\begin{array}{l}3 \\
\text { months }\end{array}$ & $\begin{array}{l}6 \\
\text { months }\end{array}$ & $\begin{array}{l}12 \\
\text { months }\end{array}$ \\
\hline \multicolumn{8}{|l|}{ Patient 1} \\
\hline & LVEDD, cm & 8.4 & 5.0 & 5.0 & & & 8.0 \\
\hline & LVESD, cm & 6.9 & 3.9 & 3.7 & & & 7.3 \\
\hline & LVEF, \% & 13 & 40 & 40 & & 30 & 10 \\
\hline & TAPSE, cm & 2.0 & 1.4 & 1.5 & & & 1.3 \\
\hline \multicolumn{8}{|l|}{ Patient 2} \\
\hline & LVEDD, $\mathrm{cm}$ & 7.4 & 4.5 & 5.0 & 5.4 & 6.1 & 5.8 \\
\hline & LVESD, $\mathrm{cm}$ & 7.0 & 3.2 & 4 & 5.2 & 5.5 & 5.4 \\
\hline & LVEF, \% & 15 & 55 & 55 & 35 & 35 & 25 \\
\hline & TAPSE, cm & & 1.5 & 1.2 & 1.9 & 1.3 & 1.4 \\
\hline \multicolumn{8}{|l|}{ Patient 3} \\
\hline & LVEDD,cm & 7.6 & 4.4 & 5.5 & 6.5 & 6.6 & 7.1 \\
\hline & LVESD, cm & 7.1 & 3.4 & 5 & 6.1 & 6.3 & 6.7 \\
\hline & LVEF, \% & 15 & 55 & 55 & 15 & 10 & 10 \\
\hline & TAPSE, cm & 1.9 & 2.0 & 2.0 & 1.5 & 1.0 & \\
\hline
\end{tabular}

LVEDD - left ventricular end-diastolic dimension

LVSD - left ventricular end-systolic dimension

LVEF - left ventricular ejection fraction

TAPSE - tricuspid annulus plane systolic excursion 


\section{Discussion}

Since the beginning of our program in 2003, a total of 256 patients have had longterm LVADs implanted. Of those patients, only 3 patients demonstrated evidence of $L V$ recovery allowing for either explantation or inactivation of their device. All patients in this case series had placement of a Heartmate II LVAD which is a continuous flow device, and no patients were treated with the selective beta agonist, clenubetrol as had been done in cited previous studies. As part of our institutions protocol, all patients in this series were continued on guideline directed medical therapy with beta blockade and ACE inhibitors that were slowly titrated to maximally tolerated dosages throughout the entire duration of their care - including prior to implantation, during implantation, and after explantation. All three patients had an ICD in place, however none met current guidelines for cardiac resynchronization therapy. Despite aggressive medical management, cardiac rehabilitation and close follow up all patients LVEF's started to decline and by 1 year all patients had developed recurrent severe LV dysfunction and significant symptoms.

The experience at our institution is consistent with the above mentioned INTERMACS recovery rate and very different from the both recovery studies with pulsatile or continuous flow LVAD, with or without clenbutirol $(1,2)$. While there is evidence that pulsatile LVADs do provide more offloading for the left ventricle we cannot equate the recovery rate to be secondary to this factor alone, given that recovery rates of similar magnitude were reproduced with continuous flow devices.

In the initial study demonstrating such pronounced rate of LV recovery, patients were treated with clenubetrol, a selective beta-2 agonist, with the goal of the medication to increase myocardial strength and contractility. Later, in a small trial by George et al (4), 7 patients were trialed on oral clenubetrol following LVAD implantation and dosages were slowly titrated up. Despite a statistically significant increase in skeletal muscle mass, a trend towards increase LV mass and a significant increase in end diastolic dimension, cardiac function did not improve.

Our experience is well aligned with data gathered from other larger datasets. We have evaluated this subject matter in a previous review in 2012 (5). At that time we evaluated several large case series of patients in an effort to decrease any random fluctuations secondary to small, single center studies and found that at most prevalence of LV recovery was $24 \%$ in these patient populations, with majority of these studies having rates of LV recovery in the single digits. In the INTERMACS database, which is composed of data from all LVAD patients, their recovery rate is similar to what we found in these other large case series at a $3 \%$ at three years (3). As INTERMACS does not have data on outcomes after LVAD explantation, we are unable to determine from their dataset if this $3 \%$ of patients went on to have sustainable, durable LV recovery following device removal or developed recurrent cardiomyopathies. As compared to INTERMACS, The United Network for Organ Sharing (UNOS) database includes only patients actively listed for transplantation, and frequently these patients tend to be younger and have less comorbidities than 
the general heart failure population. Given this population is likely more similar in demographics to the patients represented in the recovery trials, mostly young people with nonischemic cardiomyopathy of recent onset $(1,2)$, one would have expected the UNOS population to yield a recovery rate more similar to the trials, but that was not the case. In the UNOS population, only $5 \%$ of patients underwent LVAD explantation for LV recovery (7). The UNOS study did look at outcomes after explantation, however the primary end point in their analysis was mortality at one year. They did not comment on development of recurrent heart failure. Using mortality as primary end point would skew the impression that LV recovery is more durable than it really is as in our case series none of our patients would have met the primary endpoint- all were alive one year after explantation, yet all of them had developed recurrent heart failure by this point (7).

Additional evidence against complete, durable LV recovery has also been demonstrated at the cellular level. Improvement in cardiac size and function with hemodynamic unloading on LVAD is well studied. The signs include regression of cardiomyocyte hypertrophy, reduction of myocardial mass and myocyte size (6), improvement in myocyte contractility (7), , up-regulation of beta-1-adrenergic receptors, increase in their density, restoration of contractile response to betaadrenergic stimulation (8), decrease in LV dimensions and pulmonary capillary wedge pressure, increase in LVEF, cardiac index and mean aortic pressure (9), etc. On a metabolic level, there is improvement of collagen handling in the ventricle (10) and a decrease in levels of circulating and intrinsic myocardial inflammatory mediators (11). However, the normalization is usually incomplete. In the samples of myocardium before and after several months of support on LVAD, there was no difference in capillary density, cardiac fibrosis, or macrophage density. On the other hand, there was a very significant - almost 17-fold decrease in fibroblast-specific collagen expression, showing that while macroscopically there were significant changes in the LV achieved by mechanical unloading, there is persistent abnormalities on the microscopic level in regards to cell modeling and gene expression (12). Reversal of the geometric alternations by mechanical unloading does not equate to reversal of the cellular and transcriptional machinery. While the LV may appear normal on echocardiography, microscopically it is not which creates this false illusion of complete recovery (13).

Perhaps the major factor determining the rate of recovery in our case series, versus the papers by Birks et al $(1,2)$, is patient selection. In their studies majority of the patients were under the age the 50 , which is significantly younger than the typical average decompensated heart failure population. Also of note, of the 12 people who underwent device removal, 10 had carried a diagnosis of HF for less than 3 years which is a relatively short time as compared to the patients in this series in which 2 of them had carried the diagnosis for greater than 10 years. These differences are also echoed in the Texas Heart Study in which $4.1 \%$ of their LVAD patients had successful LVAD explantation for recovery, however one-third of those patients who developed LV recovery were under the age of 30 when their device was initially implanted (14). INTERMACS data also corroborates that age < 50 is an independent predictor of LV recovery, as is having a short duration from 
the time of cardiac diagnosis (<2 years), a non-ischemic etiology, LVEDD $<6.5 \mathrm{~cm}$, creatinine $<1.2 \mathrm{mg} / \mathrm{dL}$ and the absence of an ICD (15).

In general, resolution of left ventricular dysfunction following LVAD implantation allowing device removal is likely rather the exception than the rule. Younger people are more prone to have acute myocarditis, alcohol or drug related disease, or peripartum cardiomyopathy - conditions with high rate of spontaneous recovery. Myocardial offloading with LVAD simply stabilizes them and gives the time to recover.

If our experience is typical, myocardial recovery does not occur en masse on LVAD support. Hemodynamic LV unloading improves geometry and function, but the underlying process persists. When hemodynamic support is taken away, cardiomyopathy relapses within 6 months. The term "remission" is more appropriate than "recovery". 


\section{References}

1. Birks EJ, Tansley PD, Hardy J et al. Left ventricular assist device and drug therapy for the reversal of heart failure. The New England journal of medicine 2006;355:1873-84.

2. Birks EJ, George RS, Hedger M et al. Reversal of severe heart failure with a continuous-flow left ventricular assist device and pharmacological therapy: a prospective study. Circulation 2011;123:381-90.

3. Topkara VK, Garan AR, Fine B et al. Myocardial Recovery in Patients Receiving Contemporary Left Ventricular Assist Devices: Results From the Interagency Registry for Mechanically Assisted Circulatory Support (INTERMACS). Circulation Heart failure 2016;9.

4. George I, Xydas S, Mancini DM et al. Effect of clenbuterol on cardiac and skeletal muscle function during left ventricular assist device support. The Journal of heart and lung transplantation : the official publication of the International Society for Heart Transplantation 2006;25:1084-90.

5. Guglin M, Miller L. Myocardial recovery with left ventricular assist devices. Curr Treat Options Cardiovasc Med 2012;14:370-83.

6. Nakatani S, McCarthy PM, Kottke-Marchant K et al. Left ventricular echocardiographic and histologic changes: impact of chronic unloading by an implantable ventricular assist device. Journal of the American College of Cardiology 1996;27:894-901.

7. Dipla K, Mattiello JA, Jeevanandam V, Houser SR, Margulies KB. Myocyte recovery after mechanical circulatory support in humans with end-stage heart failure. Circulation 1998;97:2316-22.

8. Ogletree-Hughes ML, Stull LB, Sweet WE, Smedira NG, McCarthy PM, Moravec CS. Mechanical unloading restores beta-adrenergic responsiveness and reverses receptor downregulation in the failing human heart. Circulation 2001;104:881-6.

9. Frazier $\mathrm{OH}$, Benedict $\mathrm{CR}$, Radovancevic $\mathrm{B}$ et al. Improved left ventricular function after chronic left ventricular unloading. The Annals of thoracic surgery 1996;62:675-81; discussion 681-2.

10. Drakos SG, Kfoury AG, Selzman $\mathrm{CH}$ et al. Left ventricular assist device unloading effects on myocardial structure and function: current status of the field and call for action. Curr Opin Cardiol 2011;26:245-55.

11. Torre-Amione G, Stetson SJ, Youker KA et al. Decreased expression of tumor necrosis factor-alpha in failing human myocardium after mechanical circulatory support : A potential mechanism for cardiac recovery. Circulation 1999;100:1189-93. 
12. Farris SD, Don C, Helterline D et al. Cell-Specific Pathways Supporting Persistent Fibrosis in Heart Failure. Journal of the American College of Cardiology 2017;70:344-354.

13. Uriel N, Kim G, Burkhoff D. Myocardial Recovery After LVAD Implantation: A Vision or Simply an Illusion? Journal of the American College of Cardiology 2017;70:355-357.

14. Frazier OH, Baldwin AC, Demirozu ZT et al. Ventricular reconditioning and pump explantation in patients supported by continuous-flow left ventricular assist devices. The Journal of heart and lung transplantation : the official publication of the International Society for Heart Transplantation 2015;34:766-72.

15. Wever-Pinzon O, Drakos SG, McKellar SH et al. Cardiac Recovery During Long-Term Left Ventricular Assist Device Support. Journal of the American College of Cardiology 2016;68:1540-53. 